\title{
First-principles Calculations on Electronic and Elastic Properties of CulnS2 andCulnSe2 at Ambient Pressure
}

\author{
Chunjie Wang ${ }^{1, a}$, Xue Li ${ }^{1, b}$ and Yue Wang ${ }^{2, c^{*}}$ \\ ${ }^{1}$ College of Engineering, Bohai University, Jinzhou, 121013, China \\ ${ }^{2}$ College of New Energy, Bohai University, Jinzhou, 121013, China

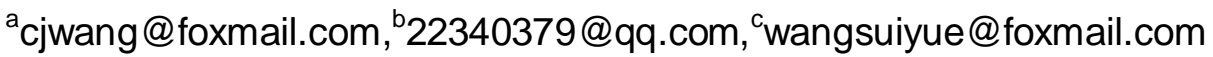

Keywords: First-principles calculation; Energy band structure; Elastic coefficient; Debye temperature.

\begin{abstract}
An investigation on energy band structures, density of states, elastic coefficients and Debye temperature of $\mathrm{CuInS}_{2}$ and $\mathrm{CuInSe}_{2}$ at ambient pressure were conducted via first-principles calculations based on density functional theory (DFT). The results show that $\mathrm{CuInS}_{2}$ and $\mathrm{CuInSe}_{2}$ are direct band gap semiconductor. The valence bands and conduction bands are mainly comes from the contributions of the $\mathrm{p}$ and $\mathrm{d}$ electrons. Moreover, the calculated results of elastic coefficients indicate that the tetragonal structures of $\mathrm{CuInS}_{2}$ and $\mathrm{CuInSe}_{2}$ are mechanically stable at ambient pressure, and their Debye temperatures are $339 \mathrm{~K}$ and $425 \mathrm{~K}$, respectively.
\end{abstract}

\section{Introduction}

The ternary compound CuInX2 $(X=S, S e)$ is a very promising thin film solar cell material. Its date of energy conversion efficiency, service life and anti-radiation ability are better than the most widely used polycrystalline and amorphous thin film solar cell.CuInS2 ( $\mathrm{X}=\mathrm{S}, \mathrm{Se}$ ) is a typical I-III-VI compounds and direct bandgap semiconductor material, with a chalcopyrite structure at normal temperature and pressure. This structure can be seen as the formation of $\mathrm{ZnS}$ units of two cubic sphalerite structures along the c-axis, except that the $\mathrm{Zn} 2+$ sites are replaced by $\mathrm{Cu}+$ ions and $\mathrm{In} 3+$. Each $\mathrm{Cu}$ atom or In atom is surrounded by four Se Atoms are bonded to form a tetrahedral structure; the same Se atoms also with the surrounding two $\mathrm{Cu}$ atoms and two In atoms into a bond to form a Se atom as the center of the tetrahedron structure, because such materials have excellent photoelectric Characteristics, so its structure and physical properties also launched a wide range of research.

High pressure has been widely concerned by researchers as one of the means to improve the performance of materials. The structural phase transition of CuInX2 $(X=S, S e)$ were studied under high pressure in the range of 6-10 GPa, both of which are transformed into $\mathrm{NaCl}$ structures. At higher pressures, $\mathrm{CuInSe} 2$ undergoes a phase transition from $\mathrm{NaCl}$ structure to orthorhombic structure, and such phase transition is irreversible. $\mathrm{CuInSe} 2$ and $\mathrm{CuInS} 2$ become sphalerite structure and disordered structure, respectively. To enhance their application functionality by tailoring the properties, it is necessary to investigate the physicochemical properties of initial structure of both materials deeply. It is well known that the elastic wave propagation effect reveals the interaction between atoms, the structural mechanics stability and the mechanism of structural transformation. In this paper, we studied the energy band structures, elasticity coefficients and Debye temperatures of $\mathrm{CuInS} 2$ and $\mathrm{CuInSe} 2$ with tetragonal phase by using the first principle with density functional theory. The main purpose of this study is to investigate the difference in the physicochemical characteristics of both materials at high pressure.

\section{Computing Method}

The first-principles calculations used in this study are based on the density functional theory, the pseudopotential method, and the Castep software package in Material Studio. The interaction 
between electrons and ions is carried out by using the super-soft pseudopotential method. Generalized gradient approximation used in the calculation is described in the form of PBE (Perdew-Berke-Ernzerhof) exchange-correlation function of the system. The reseau of point $\mathrm{k}$ in reciprocal space is $5 \times 5 \times 6$ and the plane wave truncation can be selected as $700 \mathrm{eV}$. The electronic configuration of each element in $\mathrm{CuInX}_{2}(\mathrm{X}=\mathrm{S}, \mathrm{Se})$ is as follows. $\mathrm{Cu}$ : 3d10 4s1, In: 4d10 5s2 5p1, $\mathrm{S}$ (Se): $3 \mathrm{~s} 2 \mathrm{3p} 4$. The lattice constant of tetragonal $\mathrm{CuInS}_{2}$ used in the calculation is as follows. $\mathrm{a}=$ $0.5412 \mathrm{~nm}, \mathrm{c}=1.214 \mathrm{~nm}$, space group I4-2d; The lattice constant of tetragonal $\mathrm{CuInSe}_{2}$ is as follows: $\mathrm{a}=0.5782 \mathrm{~nm}, \mathrm{c}=1.162 \mathrm{~nm}$, and space group is $\mathrm{I} 4-2 \mathrm{~d}$.

\section{Results and Discussion}

Property of Electronic Structure. The lattice constant and band gap optimization results for $\mathrm{CuInS}_{2}$ and $\mathrm{CuInSe} \mathrm{I}_{2}$ at ambient pressure are shown in Table 1. It can be seen that the error between the lattice constant and the experimental value is less than $3.5 \%$. Figure 1 (a) is the band structure of $\mathrm{CuInS}_{2}$ at ambient pressure. It can be noted from the figure that the highest point of the $\mathrm{CuInS}_{2}$ valence band and the lowest point of the conduction band are located at the point of the Brillouin zone. This fact means that $\mathrm{CuInS}_{2}$ is a direct band gap semiconductor, with bandgap width of 1.36 $\mathrm{eV}$. This result is smaller than the actual band gap of $1.5 \mathrm{eV}$, which can be due to the systematic error of the GGA theory. In this study, the scissors factor was used to correct the calculation results to solve the problem of large energy gap error in GGA calculation. The scissors factor of $\mathrm{CuInS}_{2}$ is $0.4 \mathrm{eV}$. The electron density is shown in Fig. 1 (b).

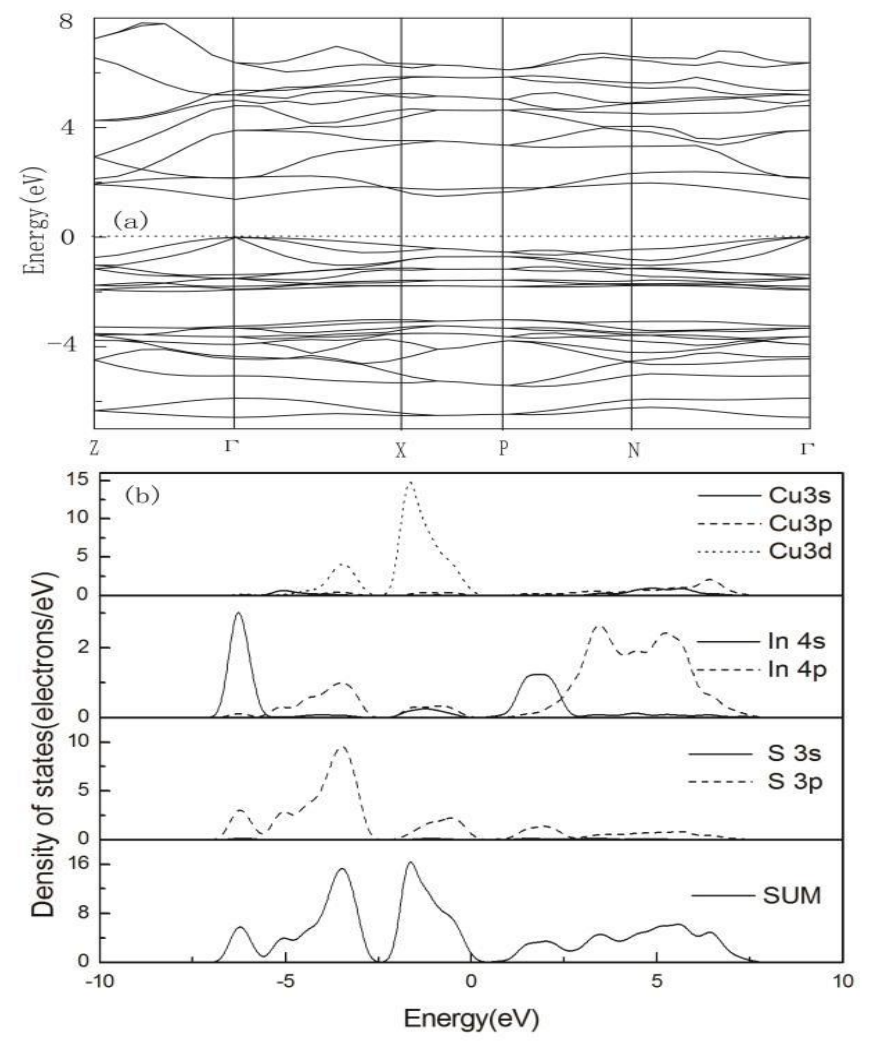

Figure 1. Energy band structures and states density of $\mathrm{CuInS}_{2}$ at ambient Pressure.

Qualitative analysis of the band near the Fermi surface indicate that the contribution to the band gap is mainly comes from the contribution of the p-state and the d-state electrons, while the contribution to the s-state electrons can be negligible. The valence band maximum up to $-7.1 \mathrm{eV}$ is mainly composed of the $3 \mathrm{~d}$ state of $\mathrm{Cu}$ and the $3 \mathrm{P}$ state of $\mathrm{S}$. The $3 \mathrm{p}$ state of $\mathrm{Cu}$, the $4 \mathrm{p}$ state of $\mathrm{In}$, and the $3 p$ state of $S$ are the conduction band energy levels above the Fermi surface.

Fig. 2 is the energy band structures and density of states of $\mathrm{CuInSe}_{2}$ at ambient Pressure. It can be seen from Fig. 2 that the valence band top and the conduction band bottom of $\mathrm{CuInSe}_{2}$ are 
located at point $\Gamma$, implying $\mathrm{CuInSe}_{2}$ is a semiconductor material with direct band gap. The band gap is $0.84 \mathrm{eV}$, which is close to the actual band gap of CuInSe 2 . Figure 2 (b) shows the electronic states density of $\mathrm{CuInSe}_{2}$ under ambient pressure. Considering the influence of electrons near the Fermi surface, the energy bands in the valence band to $-6.8 \mathrm{eV}$ are mainly comprised of hybridization of $3 \mathrm{~d}$ state of $\mathrm{Cu}$ and $4 \mathrm{p}$ state of Se.

It can be seen from the figure that the $3 p$ state of $\mathrm{Cu}$, the $4 \mathrm{p}$ state of $\mathrm{In}$, and the $3 p$ state of $\mathrm{S}$ are the conduction band energy levels above the Fermi surface. The electrons of s- state have little effect on the band near the Fermi surface. By contrast, $\mathrm{CuInS}_{2}$ and $\mathrm{CuInSe}_{2}$ are both direct band gap semiconductor materials with the band gap in the range of $1.0 \sim 1.5 \mathrm{eV}$ which can absorb more sunlight and is favorable for photoelectron transition, such performance means that $\mathrm{CuInS}_{2}$ and $\mathrm{CuInSe} \mathrm{S}_{2}$ are excellent photoelectric materials. Moreover, CuInSe $\mathrm{C}_{2}$ can easier to realize the overlap of valence band and conduction band under pressure, which can be attributed to its narrow band gap.
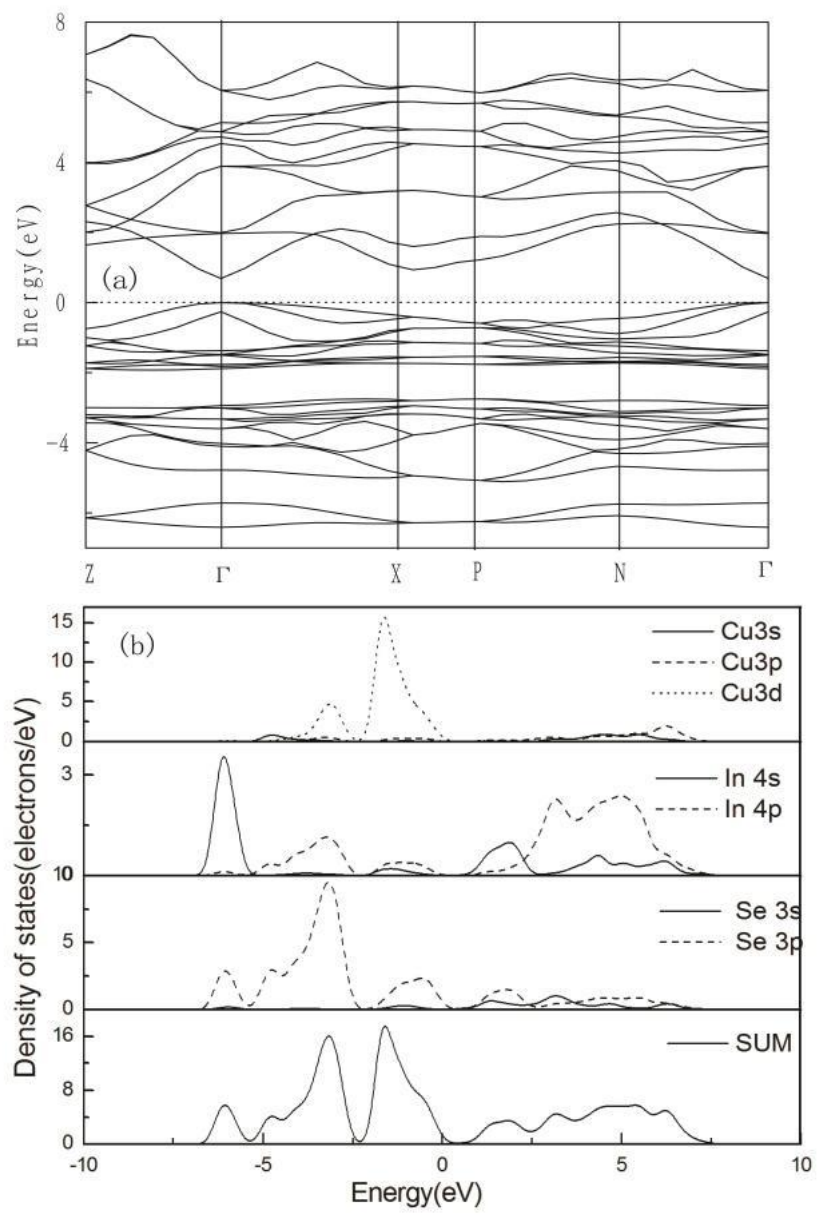

Figure 2. Energy band structures and states density of $\mathrm{CuInSe}_{2}$ at ambient Pressure

Table 1 Lattice constants and band gaps of $\mathrm{CuInS}_{2}$ and $\mathrm{CuInSe}_{2}$ at ambient pressure

\begin{tabular}{|c|c|c|c|c|}
\hline \multirow{2}{*}{ Sample } & \multicolumn{2}{|c|}{ lattice parameter/nm } & \multirow{2}{*}{ Band /eV } & \multirow{2}{*}{ Band type } \\
\cline { 2 - 3 } & $\mathrm{a}$ & $\mathrm{b}$ & 1.36 & direct \\
\hline $\mathrm{CuInS}_{2}$ & 0.5521 & 1.2174 & 0.84 & direct \\
\hline $\mathrm{CuInSe}_{2}$ & 0.5839 & 1.1734 & 0.84 \\
\hline
\end{tabular}


Elastic Coefficient and Mechanical Stability of Cell Structure. The elastic modulus is an important characteristic of crystalline material. In this study, in order to make the system reaches equilibrium, each deformation of the system is completely relaxed. The elastic modulus, bulk modulus and shear modulus of $\mathrm{CuInS}_{2}$ and $\mathrm{CuInSe}_{2}$ under normal pressure are shown in Table 2.

Table 2 Elastic coefficients Cij, bulk modulus B and shear modulus $\mathrm{G}$ of $\mathrm{CuInS}_{2}$ and $\mathrm{CuInSe}_{2}$ at ambient pressure $(\mathrm{GPa})$

\begin{tabular}{|c|c|c|c|c|c|c|c|}
\hline Sample & C11 & C33 & C44 & C12 & C13 & B & G \\
\hline CuInS $_{2}$ & 82.4 & 82.2 & 37.5 & 51.1 & 52.2 & 62.0 & 28.7 \\
\hline CuInSe $_{2}$ & 81.9 & 81.3 & 34.1 & 54.5 & 55.4 & 64.4 & 26.3 \\
\hline
\end{tabular}

$\mathrm{CuInS}_{2}$ and $\mathrm{CuInSe} \mathrm{S}_{2}$ space groups are I4-2d with tetragonal structure. For the tetragonal structure of the crystal, the structure of the mechanical stability conditions are as follows.

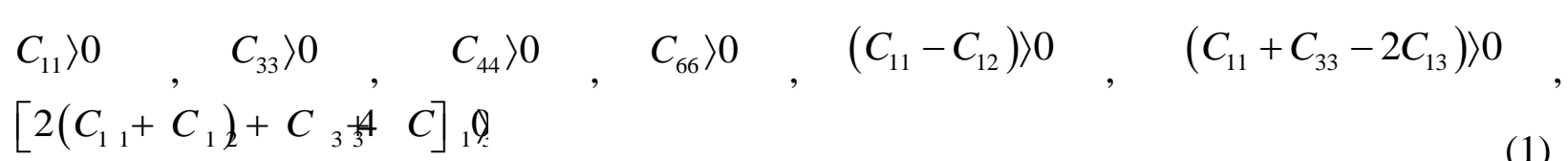

According to the data in Table 2, the elastic coefficients of $\mathrm{CuInS}_{2}$ and $\mathrm{CuInSe}_{2}$ satisfy the mechanical stability conditions, which means both materials shows stable mechanical properties under normal pressure.

Debye Temperature. Debye temperature can describe the upper limit of the phonon vibration frequency in lattice vibration, and it can be expressed as:

$$
\theta_{D}=\frac{h}{k}\left[\frac{3 n}{4 \pi}\left(\frac{N_{A} \rho}{M}\right)\right]^{1 / 3} v_{m}
$$

$\mathrm{h}$ is the Planck constant; $\mathrm{k}$ is the Boltzmann constant; $\mathrm{N}_{\mathrm{A}}$ is the Avogadro constant; $\mathrm{n}$ is the number of atoms in a molecule; $M$ is the molar mass; $\rho$ is the density; $\mathrm{Vm}$ is the average propagation velocity of the elastic wave, which has a closely relationship with the shear wave velocity (vt) and the longitudinal wave velocity (vl), and the formulas can be described as follows:

$$
\begin{aligned}
& v_{\mathrm{m}}=\left[\frac{1}{3}\left(\frac{2}{v_{\mathrm{t}}^{3}}+\frac{1}{v_{l}^{3}}\right)\right]^{-1 / 3} \\
& v_{l}=\sqrt{\frac{3 B+4 G}{3 \rho}} \\
& v_{t}=\sqrt{\frac{G}{\rho}}
\end{aligned}
$$

According to the calculation results of body elastic modulus and shear modulus, the information about shear wave velocity, longitudinal wave velocity, mean wave velocity and Debye temperature of $\mathrm{CuInS}_{2}$ and $\mathrm{CuInSe} \mathrm{I}_{2}$ are listed in Table 3. For Debye temperature, it is possible to point out the order of magnitude of the lattice vibrational frequency. The $\theta_{\mathrm{d}}$ of the crystal is more than 300 500 $\mathrm{K}$, and the upper limit of the lattice vibration frequency is $10^{13} \mathrm{~Hz}$.

Table 3 Longitudinal, transverse, average elastic wave velocities and Debye temperature of $\mathrm{CuInS}_{2}$ and $\mathrm{CuInSe} \mathrm{It}_{2}$ at ambient pressure

\begin{tabular}{|l|l|l|l|l|}
\hline Sample & $v_{l}\left(m \cdot s^{-1}\right)$ & $v_{t}\left(m \cdot s^{-1}\right)$ & $v_{m}\left(m \cdot s^{-1}\right)$ & $\theta_{d} / K$ \\
\hline $\mathrm{CuInS}_{2}$ & 8816 & 4717 & 4544 & 339 \\
\hline $\mathrm{CuInSe}$ & 4398 & 8459 & 4921 & 425 \\
\hline
\end{tabular}




\section{Conclusion}

In this paper, the Castep module in Materials Studio software were used to calculate the band structure phase transition and electron density of $\mathrm{CuInS}_{2}$ and $\mathrm{CuInSe} \mathrm{I}_{2}$ under atmospheric pressure based on the first-principles of density functional (DFT). The results show that both $\mathrm{CuInS}_{2}$ and $\mathrm{CuInSe}_{2}$ are direct band gap semiconductor materials. The energy levels near the Fermi surface are mainly composed of states of $\mathrm{p}$ and $\mathrm{d}$. The mechanical properties of $\mathrm{CuInS}_{2}$ and CuInSe $\mathrm{C}_{2}$ are stable in the chalcopyrite structure. The Debye temperatures of $\mathrm{CuInS}_{2}$ and $\mathrm{CuInSe}_{2}$ are also calculated from the elastic coefficient, the data are $339 \mathrm{~K}$ and $425 \mathrm{~K}$, respectively. Such performance indicates that $\mathrm{CuInS}_{2}$ and $\mathrm{CuInSe} \mathrm{I}_{2}$ are excellent photoelectric materials.

\section{Acknowledgments}

This work was supported by the National Natural Science Foundation of China under Grant no. 11404032.11404034; and the Foundation of Education Department of Liaoning Province no. LY2016002. Natural Science Foundation of Liaoning Province no. 20170540014, 20170540009.

\section{Reference}

[1] H. J. Lewerenz, Development of copper indium disulfide into a solar material[J].Sol energy Mater sol cells,83(2004):395-407.

[2] S.Susanne, Wide gap chalcopyrites: material properties and solar cell[J].Thin Solid Films,403-404(2002):1-8.

[3] J.Klaer, J.Bruns and R.Henninger, Effecient CuInS2 thin film solar cells prepared by sequential process [J].Semicond Sci Technol.,50(1998):1456-1458.

[4] F. Hergert, R. Hock, S. Schorr, Pentanary chalcopyrite compounds without tetragonal deformation in the heptanary system $\mathrm{Cu}(\mathrm{A} 1, \mathrm{Ga}, \mathrm{In})(\mathrm{S}, \mathrm{Se}, \mathrm{Te}) 2[\mathrm{~J}]$. Solar Energy Materials and Solar Cells, 91(2007): 44-46.

[5] S.Schorr and G.Geandier . In-situ investigation of the temperature dependent structural phase transition in CuInSe2 by synchrotron radiation[J]. Cryst. Res. Technol., 41(2006): 450-457.

[6] J.Gonzalez and C.Rincon . Optical absorption and phase transitions in CulnSe2 and CulnS2 single crystals at high pressure[J].J. Appl. Phys. , 65(1989)2031.

[7] T.Tinoco, A.Polian and D.Gomez . Structural Studies of CuInS2 and CuInSe2 under High Pressurep[J].phys. stat. sol. (b), 198 (1996): 433.

[8] D. A.Pinnick and S. A.Lee, High pressure Raman scattering study of the A1 mode of CuInS2[J]. J. Raman Spectroscopy. 2003, 34:142-144.

[9] M. D.Segall, First-principles simulation: ideas, illustrations and the CASTEP code [J].J. Phys.: Condens. Matter, 14(2002) 2717-2744.

[10] J .P.Perdew , K.Burke and M.Ernzerhof , Generalized Gradient Approximation Made Simple, [J].Phys. Rev. Lett. , 77(1996): 3865.

[11]G.Gokoglu , First Principles Study of Barium Chalcogenides[J].J Phys Chem Solids, 69( 2008) : 2924-2927.

[12] Z.J.Wu , E.J.Zhao and H.P.Xiang , Crystal Structures and Elastic Properties of Superhard IrN2 and IrN3 from First Principles [J].Phys Rev B, 76( 2007) : 054115.

[13]Z.M.Sun, S. Li and R. Ahuja , Calculated Elastic Properties of M2AlC ( $\mathrm{M}=\mathrm{Ti}, \mathrm{V}, \mathrm{Cr}$, $\mathrm{Nb}$ and Ta) [J].Solid State Commun, 129(2004 ) : 589-592. 